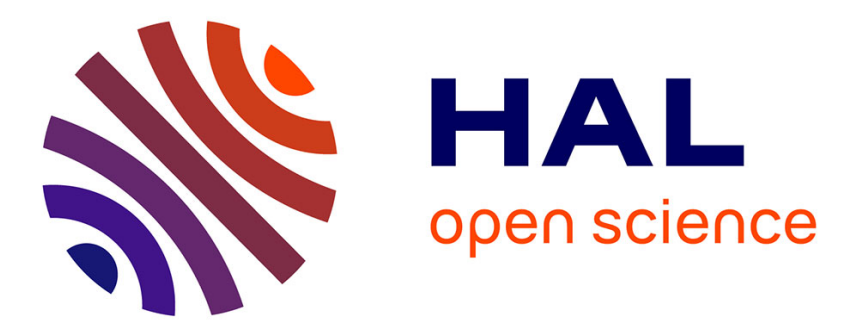

\title{
Mixing block copolymers with phospholipids at the nanoscale: from hybrid polymer/lipid worm-like micelles to vesicles presenting lipid nano-domains
}

T. P. Tuyen Dao, Annie Brûlet, Fabio Fernandes, Meriem Er-Rafik, Khalid Ferji, Jean-Paul Chapel, Ralf Schweins, Alexander Fedorov, Marc Schmutz, Manuel Prieto, et al.

\section{To cite this version:}

T. P. Tuyen Dao, Annie Brûlet, Fabio Fernandes, Meriem Er-Rafik, Khalid Ferji, et al.. Mixing block copolymers with phospholipids at the nanoscale: from hybrid polymer/lipid wormlike micelles to vesicles presenting lipid nano-domains. Langmuir, 2017, 33 (7), pp.1705-1715. 10.1021/acs.langmuir.6b04478 . hal-01448607

\section{HAL Id: hal-01448607 https://hal.science/hal-01448607}

Submitted on 30 Oct 2018

HAL is a multi-disciplinary open access archive for the deposit and dissemination of scientific research documents, whether they are published or not. The documents may come from teaching and research institutions in France or abroad, or from public or private research centers.
L'archive ouverte pluridisciplinaire HAL, est destinée au dépôt et à la diffusion de documents scientifiques de niveau recherche, publiés ou non, émanant des établissements d'enseignement et de recherche français ou étrangers, des laboratoires publics ou privés. 


\title{
Mixing block copolymers with phospholipids at the nanoscale: from hybrid polymer/lipid worm-like micelles to vesicles presenting lipid nano-domains
}

\author{
T. P. Tuyen Dao ${ }^{\mathrm{a}, \mathrm{b}, \mathrm{c}}$, A. Brûlet ${ }^{\mathrm{f}}, \mathrm{F}$. Fernandes $^{\mathrm{c}, \mathrm{d} *}$, M. Er-Rafik ${ }^{\mathrm{e}}$, K. Ferji $^{\mathrm{a}, \mathrm{b}}$, R. Schweins $^{\mathrm{g}}, \mathrm{J}-\mathrm{P}$. \\ Chapel $^{\mathrm{h}}$, A. Fedorov ${ }^{\mathrm{c}}$, M. Schmutz ${ }^{\mathrm{e}}$, M. Prieto ${ }^{\mathrm{c}}$, O. Sandre ${ }^{\mathrm{a}, \mathrm{b}}$, J-F. Le Meins ${ }^{\mathrm{a}, \mathrm{b} *}$ \\ a: University of Bordeaux, LCPO UMR 5629, 16 avenue Pey Berland, F-336oo Pessac, France \\ b: CNRS, Laboratoire de Chimie des Polymères Organiques, UMR 5629, F-3360o, Pessac, France \\ c: Centro de Química-Física Molecular and Institute of Nanoscience and Nanotechnology, Universidade de Lisboa \\ Instituto Superior Técnico, 1049-001 Lisboa, Portugal \\ d: Research Unit on Applied Molecular Biosciences-Rede de Química e Tecnologia (UCIBIO-REQUIMTE), \\ Departamento de Química, Faculdade de Ciências e Tecnologia, Universidade Nova de Lisboa, Caparica, Portugal \\ e: Institut Charles Sadron, UPR 22 CNRS, Université de Strasbourg, 23 rue du Loess, 67034 Strasbourg, France \\ f: Laboratoire Léon Brillouin, UMR12 CEA-CNRS, CEA Saclay, F-91191 Gif-sur-Yvette Cedex, France \\ g: ILL Grenoble, DS LSS, CS20156, 71 Ave Martyrs, F-38042 Grenoble 9, France \\ h: Univ Bordeaux, CNRS, Ctr Rech Paul Pascal CRPP, UPR 8641, F-336oo Pessac, France
}

KEYWORDS: Hybrid Polymer Lipid Unilamellar Vesicle, lipid nanodomains, membrane, polymersomes

\begin{abstract}
Hybrid, i.e. intimately mixed polymer/phospholipid vesicles can potentially marry in a single membrane the best characteristics of the two separate components. The ability of amphiphilic copolymers and phospholipids to selfassemble into hybrid membranes has been studied until now at the sub-micron scale using optical microscopy on Giant Hybrid Unilamellar Vesicles (GHUVs), but limited information is available on Large Hybrid Unilamellar Vesicles (LHUVs). In this work, copolymers based on poly(dimethyl siloxane) and poly(ethylene oxide) with different molar masses and architectures (graft, triblock) were associated with 1,2-di-palmitoyl-sn-glycero-3-phosphocholine (DPPC). Classical protocols of LUV formation were used to obtain nano-sized self-assembled structures. Using Small Angle Neutron Scattering (SANS), Time Resolved Förster Resonance Energy Transfer (TR-FRET) and Cryo-Transmission Electron Microscopy (Cryo-TEM), we show that copolymer architecture and molar mass have a direct consequence on the formation of hybrid nanostructures that can range from worm-like hybrid micelles to hybrid vesicles presenting small lipid nanodomains.
\end{abstract}

Hybrid polymer/phospholipid vesicles are emerging structures that combine the biocompatibility and biofunctionality of liposomes, with the robustness, low permeability, and functional variability conferred by copolymer chains. This should become of great interest in pharmaceutical applications for which a few formulations based on liposomes are commercially available despite decades of research, viz. DaunoXome ${ }^{\circledR}$, Doxil ${ }^{\circledR} /$ Caelyx $^{\circledR}$, Thermodox ${ }^{\circledR}$, Visudyne ${ }^{\circledR}$.

The literature on the subject is still relatively limited,1-2 although the scientific output is increasing with growing interest from different scientific communities (biophysicists, biologists, physicochemists). Most of the knowledge obtained on their membrane structure and properties has been acquired through experiments made on Giant Hybrid Unilamellar Vesicles (GHUVs, whose characteristic size is a few tens of microns) which are commonly obtained by the electroformation technique, although alternative protocols have been also reported. ${ }^{3-4}$ Literature on nanometric Large Hybrid Unilamellar Vesicles (LHUVs, whose characteristic size is around $100 \mathrm{~nm}$ ) is scarce. ${ }^{5-9}$ Most of the work available focuses on properties related to the nanostructure: permeability, ${ }^{8}$ drug release, $, 6,10$ drug targeting and cellular recognition ability ${ }^{7,11}$. LUHVs were reported by the co-assembly of phospholipids with block copolymers with a hydrophobic part based on poly(dimethylsiloxane) or poly(butadiene), formulated by processes based on film rehydration and extrusion (or sonication) ${ }^{12}$ 
techniques to obtain well-defined ,LHUVs. Very recently poly(oligoethylene glycol acrylate)- $b$ poly(lauryl acrylate) (POEGA-PLA) block copolymers have been also investigated and interacted with biological cells. ${ }^{12}$ However, detailed information about the hybrid character of the membrane and their structuration at the nanoscale are not available yet.

Globally, molecular factors governing the phase separation in these hybrid copolymer/lipid membranes are only partially understood. In addition to the expected chemical incompatibility between copolymer chains and phospholipids, one also has to consider their respective dimensions. Hydrophobic mismatch, in analogy to multicomponent lipid membranes, has been shown to play a role in the formation of stable domain in GHUVs. ${ }^{13-14}$ At the nanoscale, our group has recently provided evidence of phase separation inside the membrane of LHUVs. ${ }^{15}$ Moreover, results obtained on LHUVs ${ }^{15}$ and GHUVs ${ }^{13}$ obtained from similar polymer/lipid mixtures suggest that the lateral structures obtained on GHUVs do not reflect entirely the lateral structures obtained from LHUVs (and vice versa). Therefore, many questions remain open regarding LHUVs: What type of membrane structure do they form? Are the phospholipids homogeneously dispersed in the polymer phase or do they form nanodomains? How to tune these features, and finally can we obtain LHUVs whatever the molar mass and structure of the block copolymer used, through the classical formulation processes described previously? We propose to tackle these key issues by working on the design of LHUVs composed of 1,2-di-palmitoyl-sn-glycero-3phosphocholine (DPPC) and a series of grafted and triblock copolymer based on polydimethylsiloxane (PDMS) and polyethyleneoxide (PEO) able to form polymersomes with a membrane thickness ranging from $5 \mathrm{~nm}$ to $12 \mathrm{~nm} .{ }^{15}$ Different information about nanostructures in terms of morphology, hybrid character and distribution of the components within the membrane were acquired through common techniques to study phase separation at the nanoscale in lipid LUVs, 16 17-20 such as Time Resolved Förster resonance energy transfer (TRFRET), Cryo-Transmission Microscopy (Cryo-TEM) and Small Angle Neutron Scattering (SANS). The guidelines of the methodology and experimental procedures are described in the experimental section.

\section{EXPERIMENTAL SECTION}

Copolymers: in order to discriminate between the influence of copolymer architecture and molar mass respectively on the formation of hybrid vesicles and on their membrane structure, we used a grafted copolymer with a flexible poly(dimethylsiloxane) backbone and two poly(ethylene oxide) pendant moieties (PDMS- $g$ $\left.(\mathrm{PEO})_{2}\right)$ of $\mathrm{Mn}=2700 \mathrm{~g} / \mathrm{mol}$. This copolymer is well known to spontaneously form vesicles with a membrane thickness $(\delta \sim 5 \mathrm{~nm})$, close to the liposomes ones $(\delta \sim 3-4 \mathrm{~nm})$. We also used triblock copolymers PEO- $b$-PDMS- $b$-PEO synthesized by a block coupling approach, described in Supp. Info (S2.1). These copolymers are able to form polymersomes with a membrane thickness ranging from 5 to $12 \mathrm{~nm}$ as measured by SANS and CryoTEM (Table 1).

Lipids: DPPC, (1, 2-dipalmitoyl-sn-glycero-3phosphocholine) was chosen in order to evaluate the influence of fluidity on the membrane structure, by working below and above its melting temperature $\left(\mathrm{Tm} \sim 41^{\circ} \mathrm{C}\right)$. Fully double chain deuterated DPPC- $\mathrm{d}_{62} \quad\left(1,2\right.$-dipalmitoyl- $\mathrm{d}_{62}-\mathrm{sn}$ glycero-3-phosphocholine) was used to prepare samples for SANS measurements. DOPE-Rhod(1,2dioleoyl-sn-glycero-3-phosphoethanolamine-N-

(lissamine rhodamine B sulfonyl)) was used as an acceptor probe for TR-FRET experiments. All lipids were obtained from Avanti Polar Lipids Inc. (Alabaster, AL, Canada). DPH (1,6-diphenyl-1,3,5hexatriene) used for fluorescence anisotropy measurement was purchased from Molecular Probes (Leiden, The Netherlands).

All the vesicles were prepared by the film rehydration-extrusion method, using $100 \mathrm{~nm}$ polycarbonate filters. In some cases, the emulsionevaporation method followed by extrusion was used instead (SI S2.2.2). Hybrid vesicles were investigated in a polymer composition range from $70 \%$ to $85 \% \mathrm{w} / \mathrm{w}$ and analyzed with a whole panel of complementary techniques: static light scattering (SLS), dynamic light scattering (DLS), small angle neutron scattering (SANS), fluorescence spectroscopy (TR-FRET) and Cryo-TEM.

Small Angle Neutron Scattering: The SANS experiments were carried out at D11 small angle spectrometer at Institut Laue-Langevin (ILL), Grenoble, France. A small part of the experiments were carried out at PACE small angle spectrometer at Laboratoire Léon Brillouin (LLB) CEA, Saclay. France. Details about the methodology, measurements as well as data analysis are available in ESI 
(S2.4). In brief, we used the contrast variation technique (mixtures of $\mathrm{D}_{2} \mathrm{O} / \mathrm{H}_{2} \mathrm{O}$ of $9 / 91$ or $81 / 19$ ) to detect selectively the signal of either the lipid or the polymer phase, or to observe both (pure $\mathrm{D}_{2} \mathrm{O}$ ). For instance in lipid matching (polymer contrast), if vesicles were obtained with lipid homogenously dispersed in the polymer phase, data should be fitted by a simple vesicle form factor. On the contrary if lipid domains were present, they would appear as holes in the membrane in polymer contrast, and simple vesicle form factor should not fit the data. The SANS data analysis was done by trying different models to fit the scattered intensity curves of the samples. Various form factors were compared to data: vesicles, disks or core-shell cylinders, using the SasView program (http://www.sasview.org/). To describe the scattering of phase separated polymer/lipid vesicles (i.e. presence of lipid domains in the polymer membrane), we developed a new model based on the holey shell form factor introduced by Bergstrom et al. in their SANS study of catanionic vesicles with perforated membranes. ${ }^{28}$ This model is detailed in the section called 'hybrid vesicle' Form Factor at the end of the article.

Fluorescence spectroscopy: To probe phase separation the nanometer scale between the lipid and polymer phases, we used Forster resonance energy transfer (FRET) commonly used to detect and characterize lateral membrane domains presenting sizes smaller than 50-100 nm. Details of measurements and methods are available in ESI S.2.5. In brief, the (PDMS- $g$-(PEO) 2 ) was chemically modified with (succinimidyl 6-(N-(7-nitrobenz-2-oxa1,3-diazol-4-yl)amino)hexanoate) (NBD), as previously described, 15 and used as a donor probe for FRET experiments whereas DOPE-Rhod (1,2dioleoyl-sn-glycero-3-phosphoethanolamine-N-

(lissamine rhodamine B sulfonyl)) was used as acceptor probe. The donor probe is expected to be mainly in the polymer phase and acceptor probe mainly in the lipid phase. Their exact repartition were determined through the measurement of partition coefficient determined spectroscopically through changes in fluorescence intensity. FRET efficiencies were calculated from NBD fluorescence lifetimes in the presence and in absence of acceptors. The values obtained were therefore compared to those predicted in the case of a homogenous distribution of the components (equivalent to domain sizes below 5-10nm) and infinite phase separation, equivalent to domain sizes larger than 25$50 \mathrm{~nm}$ (or 5-10 times $R_{0}$, the Förster radius for this donor-acceptor pair, which is $5 \mathrm{~nm}$ ). Values of the area per polymer chain in a vesicle estimated from SLS and Langmuir compression isotherm measurements (See Supp. Info. section S2.3 Table S2 and S2.7) and area per lipid from literature were used for the simulations of FRET efficiency.

Cryo-TEM: Observations were performed on vitrified samples from solutions at room temperature or at $45^{\circ} \mathrm{C}$. Details about the procedure are available in ESI, Section S2.6

The global methology was the following, first, the ability of the block copolymer synthesised to form vesicle was checked by scattering techniques. Therefore a qualitative analysis of SANS curve of polymer /lipid mixtures at different temperature was made to evaluate the hybrid character of the vesicles formed. Efficiency of mixing and membrane structuration were therefore evaluated by comparing the morphology predicted by SANS via fitting procedure and those predicted by FRET experiments. The CryoTEM was used to complte the analysis and confirm conclusion brought by FRET and SANS.

\section{RESULTS AND DISCUSSION}

In a first part of the work, the vesicle forming ability of the synthesized copolymers was checked by DLS, SLS, SANS, and Cryo-TEM. All the SANS curves of suspensions of pure compounds display the characteristic $q^{-2}$ scaling law over a wide intermediate $q$ wavevector range: they are well fitted with a polydisperse vesicle (spherical shell) form factor (see Fig S11). The best fitting parameters are reported in Table 1. All copolymers form vesicles with relatively high size dispersity. Despite this fact, the membrane thickness can be accurately determined from the fit: it varies from $5.5 \mathrm{~nm}$ to $11.7 \mathrm{~nm}$ and scales with molar mas as $\mathrm{M}^{0.66}$, in good agreement with published results obtained with PMOXA- $b$-PDMS- $b$-PMOXA copolymers (Figure S30). ${ }^{21}$ These morphologies do not change when increasing the temperature from $20^{\circ} \mathrm{C}$ up to $47^{\circ} \mathrm{C}$ (Table 1). DPPC- $\mathrm{d}_{62}$ vesicles are also rather well defined: the values of membrane thickness are in good agreement with those determined by Nagle et al. by combination of SAXS with a volumetric (densitometry) study, ${ }^{22-23}$ respectively $4.57 \mathrm{~nm}$ at $20^{\circ} \mathrm{C}$ for the bilayer of DPPC in the gel $L_{\beta}$ phase and $3.51 \mathrm{~nm}$ at $50^{\circ} \mathrm{C}$ in the fluid $L_{\alpha}$ phase. A strong signature change upon main chain melting transition of lipids is observed (Fig S13). 


\section{General features of SANS curves of mixed systems}

We evaluated the behavior of the mixed polymer/lipid structures below and above the main chain melting temperature of DPPC. Experiments were done in polymer contrast, full contrast and lipid contrast. Polymer and full contrasts give almost similar curves, slightly higher for full contrast due to moderate amount of DPPC- $\mathrm{d}_{62}$ and a scattering length density close to the one of $\mathrm{D}_{2} \mathrm{O}$. Only polymer contrast data are presented in the main text of the article for clarity. As expected, the curves in lipid contrast are those which are the most affected by the change of the lipid state, (See Supp. Info Fig S13) while the effects on the curves in polymer contrast (Figure 1) are less pronounced although clearly present.

The structural changes observed in polymer contrast conditions are particularly interesting: they vary with the copolymer. Typical examples for each series of copolymers are shown in Figure 1. For the grafted copolymer, $\mathrm{PDMS}_{26}$-g- $\left(\mathrm{PEO}_{12}\right)_{2}$, the curves are changing in the intermediate $q$ range, $10^{-2}$ to $5 \cdot 10^{-2} \AA^{-1}$ (see Fig 1-a).

\begin{tabular}{|c|c|c|c|c|c|c|c|}
\hline \multirow{3}{*}{ Copolymer } & \multirow{3}{*}{$T$} & \multicolumn{5}{|c|}{ SANS } & Cryo-TEM \\
\hline & & Guinier plot & \multicolumn{4}{|c|}{ Vesicle form factor } & \multirow[b]{2}{*}{$\delta_{\mathrm{v}}(\mathrm{nm})$} \\
\hline & & $\begin{array}{c}R_{\mathrm{g}} \\
(\mathrm{nm})\end{array}$ & $\begin{array}{c}R_{\mathrm{v}} \\
(\mathrm{nm})\end{array}$ & $\sigma_{R_{\mathrm{v}}}$ & $\begin{array}{c}\delta_{\mathrm{v}} \\
(\mathrm{nm})\end{array}$ & $\sigma_{\delta_{v}}$ & \\
\hline$P D M S_{26}-g-\left(P E O_{12}\right)_{2}$ & $20^{\circ} \mathrm{C} / 47^{\circ} \mathrm{C}$ & $50 \pm 2 / 50 \pm 2$ & $36.5 / 38$ & $0.3 / 0.28$ & $5 \cdot 6 / 5 \cdot 7$ & $0.18 / 0.18$ & $5.4 \pm 0.6$ \\
\hline$P E O_{8}-b-P D M S_{22}-b-P E O_{8}$ & $20^{\circ} \mathrm{C} / 47^{\circ} \mathrm{C}$ & \multicolumn{3}{|c|}{ No Guinier Range } & $5.6 / 5.4$ & $0.14 / 0.15$ & $5.4 \pm 0.4$ \\
\hline$P E O_{12}-b-P D M S_{43}-b-P E O_{12}$ & $20^{\circ} \mathrm{C} / 47^{\circ} \mathrm{C}$ & $40 \pm 2 / 40 \pm 2$ & $16 / 16$ & $0.5 / 0.5$ & $8.8 / 8.8$ & $0.16 / 0.12$ & $8.8 \pm 0.5$ \\
\hline$P E O_{17}-b-P D M S_{67}-b-P E O_{17}$ & $20^{\circ} \mathrm{C} / 47^{\circ} \mathrm{C}$ & $47 \pm 2 / 46 \pm 2$ & $27 / 29$ & $0.45 / 0.42$ & $11.7 / 11.5$ & $0.15 / 0.15$ & $11.2 \pm 1.2$ \\
\hline$D P P C-d_{62}$ & $20^{\circ} \mathrm{C} / 47^{\circ} \mathrm{C}$ & $45 \pm 2 / 50 \pm 2$ & $27 / 31$ & $0.32 / 0.31$ & $4 \cdot 3 / 3.1$ & $0.15 / 0.15$ & $6.2 \pm 0.4 / 5 \cdot 9+0.6$ \\
\hline
\end{tabular}

Table 1 : Characteristics of LUVs of pure compounds obtained from SANS and Cryo-TEM. $R_{\mathrm{g}}$ is calculated from Guinier plot obtained with light scattering. Best fit parameters of the vesicle model (median inner radius of vesicles $\left(R_{\mathrm{v}}\right)$, its lognormal distribution width parameter $\sigma_{R v}$, median membrane thickness $\left(\delta_{v}\right)$, and its lognormal distribution $\left.\sigma_{v}\right)$ for water suspensions of pure compounds (block copolymers in $\mathrm{D}_{2} \mathrm{O}$ and deuterated lipid DPPC- $\mathrm{d}_{62}$ in $9 \% \mathrm{D}_{2} \mathrm{O}$ ). For the fits, the volume fractions of vesicles were fixed to the ones of the samples $(\Phi \sim 0.0035)$ except for $\mathrm{PEO}_{17}-b-\mathrm{PDMS}_{67}-b-\mathrm{PEO}_{17}$, which was better fitted with a lower fraction. $(\Phi \sim 0.0018)$

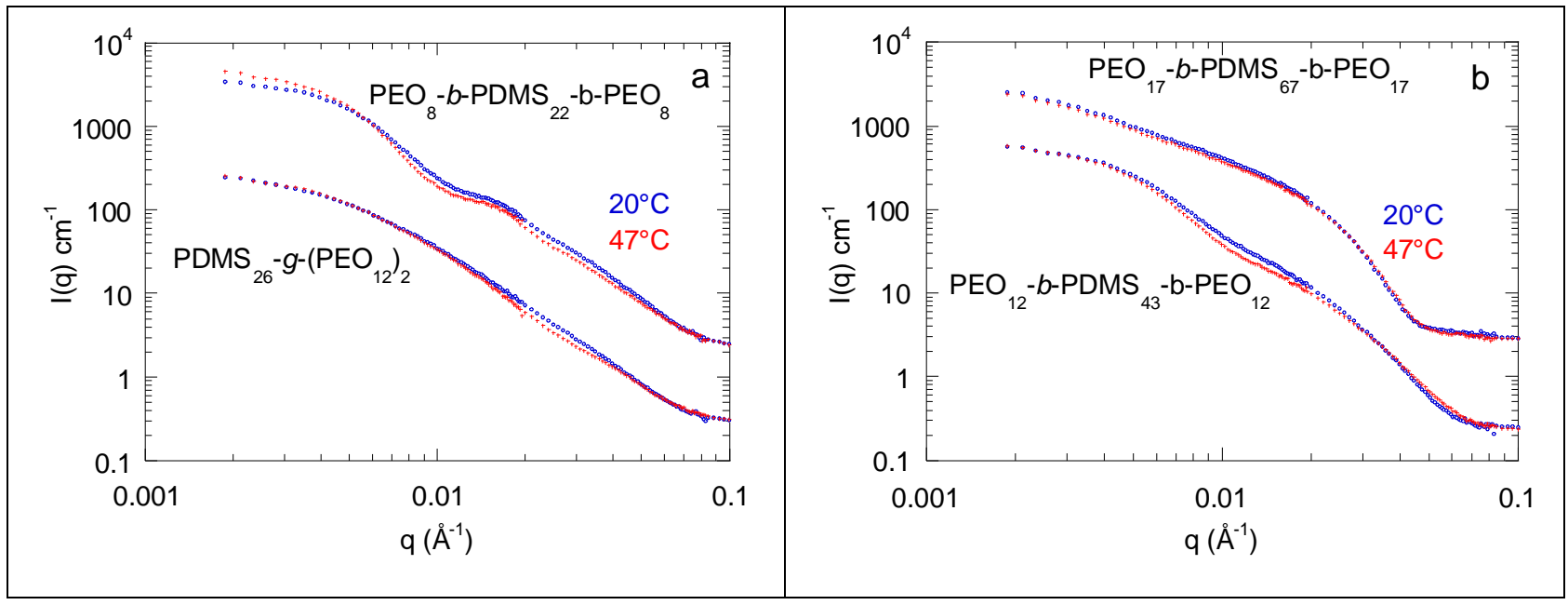

Figure 1. SANS in polymer contrast at $20^{\circ} \mathrm{C}(\mathrm{o})$ and $47^{\circ} \mathrm{C}(+)$ of mixture with a lipid fraction $\mathrm{f}_{\mathrm{d}}=0.15$ (weight fraction) (a) for two structures graft $\mathrm{PDMS}_{26^{-}} g-\left(\mathrm{PEO}_{12}\right)_{2} /$ DPPC- $\mathrm{d}_{62}$ and triblock $\mathrm{PEO}_{8}-b-\mathrm{PDMS}_{22}-b-\mathrm{PEO}_{8} / \mathrm{DPPC}_{\mathrm{d}} \mathrm{d}_{62}$ mixture, (b) for a triblock structure and two molar masses $\mathrm{PEO}_{12}-b-\mathrm{PDMS}_{43}-b-\mathrm{PEO}_{12} / \mathrm{DPPC}-\mathrm{d}_{62}$ mixture and $\mathrm{PEO}_{17}-b-\mathrm{PDMS}_{67}-b-\mathrm{PEO}_{17} / \mathrm{DPPC}_{\mathrm{d}} \mathrm{d}_{62}$ mixture. On each graph, one curve was shifted for clarity by one order of magnitude.

For the smallest PDMS triblock, (Fig 1-a), differences are visible at low $q$, in the Guinier regime of the determination of the radius of gyration, and in the intermediate $\mathrm{q}$ range where an oscillation is 
visible, up to about $5 \cdot 10^{-2} \AA^{-1}$ as for the previous copolymer. For the triblock $\mathrm{PEO}_{12}-b-\mathrm{PDMS}_{43}-b$ $\mathrm{PEO}_{12}$ (Fig 1-b), a modification

of scattering profile is observed only in the intermediate oscillation $q$ range.

The case of the largest molar mass triblock $\mathrm{PEO}_{17^{-}}$ $b$-PDMS $67-b$ - PEO $_{17}$ (Fig 1-b), is peculiar as a $q^{-1}$ decrease characteristic of an elongated scattering object is observed rather than a $q^{-2}$ scaling law characteristic of vesicles. Moreover, the evolution with temperature is almost negligible. It is important to note that the structural changes observed are completely reversible as the scattering curves at $25^{\circ} \mathrm{C}$ obtained before and after experiments at $47^{\circ} \mathrm{C}$ are completely superimposed whatever the contrast chosen, (Supp. Info Figure S17)). In polymer contrast, the two phenomena, i.e. change upon temperature of scattering curves and reversibility, are very important. Indeed, since the polymer is not sensitive to temperature, the alterations prove that polymers and lipids are somehow mixed within the vesicular structures, and the reversibility confirms that the structures formed are stable enough to follow the local changes induced by the main chain melting transition of the lipid phase with temperature.

\section{Hybrid $\quad$ PEO $_{17}-b-$ PDMS $_{67}-b-$ PEO $_{17} /$ DPPC nanostructures}

We first focus on the copolymer presenting a $q^{-1}$ dependence of the scattering intensity. Interestingly data in polymer contrast could be fitted with a core shell cylinder model, with polymers forming the core of long cylinders with a core radius around 7-8 $\mathrm{nm}$ influenced neither by temperature nor by lipid fraction. Fit parameters are indicated in Table 2. In lipid contrast, (Figure S18) we observe at high $q$ a clear and well pronounced bump that does not arise from the scattering of the vesicle membrane but rather from a well-defined shell of core shell cylindrical scattering objects. However, at low $q$, the scattering intensity especially for the lipid weight fractions $\left(f_{\mathrm{d}}=0.21\right)$ displays the oscillation characteristic of vesicles radii. These observations indicate clearly that a part of the lipid phase is mixed somehow with the copolymer in cylindrically shaped objects, whereas another part forms vesicles probably of pure lipid. At large $q$, the data could be reasonably fitted with a core-shell cylinder model and the corresponding best fit parameters are reported in Table 2 . In all these mixtures, the shell thickness $t_{\text {shell }}$ is $2 \mathrm{~nm}$, about half of the membrane of pure lipid vesicles. Interesting- ly, there is a reasonable agreement between core radii found in polymer and lipid contrasts. From these fits, it seems that a large part of the nanostructure formed presents a core-shell cylindrical shape, with lipids forming the shell around the polymer core. Such transverse phase separation in a vesicle bilayer driven by different spontaneous curvatures was predicted long time ago for a binary mixture of membrane forming molecules. ${ }^{24}$ However, it is rather counterintuitive here that the shortest chains (those of the lipids) form the outer shell, while the copolymer forms the inner core of the cylinder.

Considering the complexity of these mixtures and the presence of pure vesicles not accounted in the fits, it is not possible to discuss the variations of the other parameters observed as a function of the lipid fraction or temperature. We have also fitted the data in lipid contrast with a sum of two form factors (vesicle + core shell cylinder) as described in Supp. Info S.2.4, Fig S20, Table S7, which led to similar conclusions.

\begin{tabular}{|c|c|c|c|c|c|c|}
\hline \multirow{3}{*}{$\begin{array}{l}\text { Dispersed } \\
\text { phase } \\
\text { (lipid) } \\
\text { tion } f_{d}\end{array}$} & \multirow{3}{*}{$T$} & \multirow{2}{*}{\multicolumn{2}{|c|}{$\begin{array}{c}\text { Polymer } \\
\text { contrast }\end{array}$}} & \multicolumn{3}{|c|}{ Lipid contrast } \\
\hline & & & & \multicolumn{3}{|c|}{$\begin{array}{l}\text { Core-shell cylinder } \\
\text { model with lipid shell }\end{array}$} \\
\hline & & $\begin{array}{l}R_{\text {core }} \\
(\mathrm{nm})\end{array}$ & $\sigma_{R_{\text {core }}}$ & $\begin{array}{l}R_{\text {core }} \\
(\mathrm{nm})\end{array}$ & $\sigma_{R_{\text {core }}}$ & $\begin{array}{l}t_{\text {shell }} \\
(\mathrm{nm})\end{array}$ \\
\hline \multirow{2}{*}{0.15} & $\begin{array}{r}20^{\circ} \\
C\end{array}$ & $7 \cdot 7$ & 0.16 & 6.3 & 0.15 & 2.1 \\
\hline & $\begin{array}{r}47^{\circ} \\
C\end{array}$ & $7 \cdot 4$ & 0.17 & $5 \cdot 9$ & 0.14 & 2.0 \\
\hline \multirow{2}{*}{0.21} & $\begin{array}{r}20^{\circ} \\
C\end{array}$ & 8.6 & 0.2 & $5 \cdot 9$ & 0.16 & 2.1 \\
\hline & $\begin{array}{r}47^{\circ} \\
C\end{array}$ & 8.7 & 0.24 & 5.5 & 0.18 & 1.9 \\
\hline \multirow{2}{*}{0.3} & $\begin{array}{r}20^{\circ} \\
C\end{array}$ & 6.8 & 0.2 & 5.4 & 0.18 & 2.1 \\
\hline & $\begin{array}{c}47^{\circ} \\
C\end{array}$ & 7.1 & 0.19 & 5.1 & 0.2 & 2.0 \\
\hline
\end{tabular}

Table 2. Fit parameters obtained from the core-shell cylinder model deduced from SANS curves of $\mathrm{PEO}_{17}-b-\mathrm{PDMS}_{67}-b$ $\mathrm{PEO}_{17} /$ DPPC- $\mathrm{d}_{62}$ mixtures.

Cryo-TEM experiments indeed reveal the presence of multiple morphologies (statistics can be consulted in supporting information, Figures S34S37). This technique has already been used to observe hybrid vesicles composed of PDMS- $b$ PMOXA, DMPC and cholesterol, and rounded vesicles of different membrane thicknesses were ob- 
served but no lipid domains could be evidenced. ${ }^{5}$ In our case, faceted vesicles with a relatively thin membrane $(\sim 6 \mathrm{~nm})$ similar to those measured for pure DPPC vesicles are observed, as well as polymersomes with thick membrane $(\sim 12 \mathrm{~nm})$. Worm-like micelles are also observed and represent $\sim 50 \%$ of the population. Some of these wormlike micelles present a 'pan-like' shape, which corresponds to a worm-like micelle ending with a rounded disk. Very interestingly, the Cryo-TEM signature of a flat DPPC lipid domain in a thick polymersome membrane is visible as illustrated in the inset of Figure 2. PDMS and Phosphorous electronic contrast are similar, therefore detecting lipid phase is extremely difficult. Although some vesicles could have hybrid character non visible by Cryo-TEM, considering the amount of hybrid wormlike micelles and faceted DPPC vesicles, the number of LHUVs obtained with this high membrane thickness copolymer $(\sim 11-12 \mathrm{~nm})$ is probably limited. Wormlike micelles have been also observed very recently for mixtures composed of POPC and diblock copolymer poly(butadiene-bethylene oxide) $\left(\mathrm{PBd}_{22}-b\right.$-PEO $\left.{ }_{14}\right)$ at $29 \% \mathrm{w} / \mathrm{w}$ lipid in hybrid vesicles, using classical rehydration /extrusion technique. ${ }^{25}$

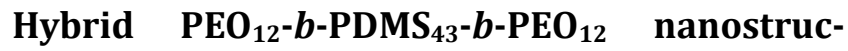 tures}

We considered a mixture of DPPC with the copolymer forming vesicle with slightly lower membrane thickness $(\sim 8 \mathrm{~nm}), \quad \mathrm{PEO}_{12}-b$-PDMS $43-b$ $\mathrm{PEO}_{12}$. In that case, a $q^{-2}$ dependence, classically observed in vesicular structure is obtained for both polymer and lipid contrast samples. We then tried to get more insight into the structure obtained, using different form factors to fit the data. In polymer contrast, we tried in particular the novel 'hybrid vesicle' model presented in the last section before the conclusions, in which lipid domains in the polymer membrane are matched, and therefore accounted as holes.

Fit works reasonably well but the parameters obtained were not physically realistic: hole size close to $1 \mathrm{~nm}$ with huge size dispersity (data not shown). Better results were obtained using a simple vesicle model as illustrated in Fig 3. Other fits and corresponding parameters can be consulted in supporting information. (Table S6 Figure S15).

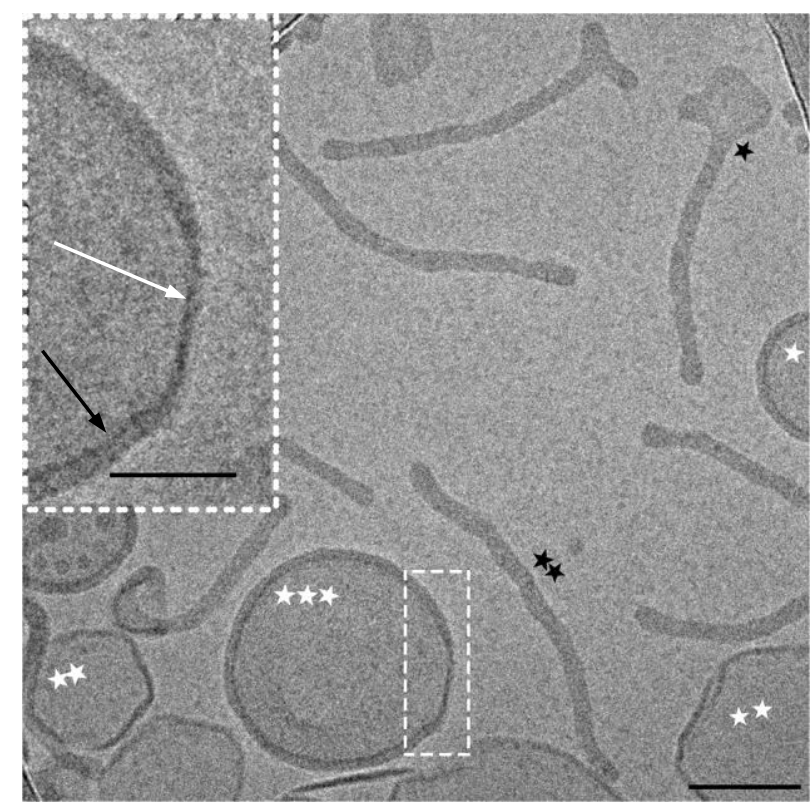

Figure 2. Cryo-TEM picture of $70 / 30$ weight ratio $\mathrm{PEO}_{17}-b$ $\mathrm{PDMS}_{67}-b-\mathrm{PEO}_{17} / \mathrm{DPPC}$ mixture quenched from room temperature. The object pointed by a black star is called "panlike" micelle. A worm-like micelle is indicated by two black stars. Vesicles can easily be identified by the thickness of their membrane; vesicle with one white star corresponds to a pure polymersome (membrane thickness $\delta_{\mathrm{v}}$ is about $11.2 \mathrm{~nm}$ ). The faceted vesicle with two white stars represents a typical pure DPPC vesicle with the thickness of $6.2 \mathrm{~nm}$. The vesicle with 3 white stars is a hybrid vesicle. In the inset, the enlargement of a hybrid vesicle is shown. The white arrow points to the thinner membrane corresponding to the lipid membrane and the black arrow points to thicker membrane corresponding to the polymer membrane. Scale bar length is $100 \mathrm{~nm}$ in the main image and $40 \mathrm{~nm}$ in the enlargement.

Globally the vesicle radius obtained by the fit is rather low with important size dispersity. It is interesting to note that taking into account this dispersity, a weight-average radius $R_{\mathrm{w}}$, can be obtained from the radius of the fit $R_{0}$ and the dispersion width $\left(R_{\mathrm{w}}=R_{0} e^{7 / 2} \sigma^{2}\right)$, in excellent agreement with the radius of gyration $R_{\mathrm{g}}$ obtained via Guinier plot. Values of membrane thickness are however well defined with a rather low dispersity. A slight thinning of the membrane is observed when temperature increases, and also when lipid fraction increases, revealing that lipids are mixed to some extent with the copolymers inside the membrane. Regarding the scattering curves in lipid contrast, good fits are also obtained with the vesicle form factor, (Figure S16) with similar vesicle radius presenting high dispersity but again with well-defined membrane thicknesses $(\sim 4 \mathrm{~nm})$, which decreases slightly with temperature in agreement with what is expected for DPPC membranes. ${ }^{22-23}$ Although the polymer and lipid may not be totally mixed, it seems from the SANS re- 
sults that there is no clear phase separation inside the membrane and that existing hybrid vesicles present a homogenous distribution of their components.

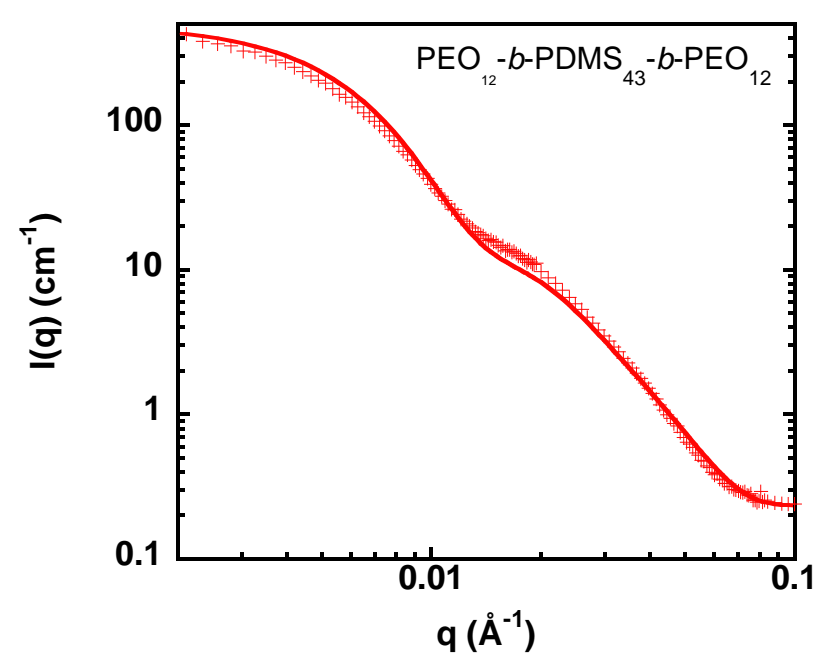

Figure 3. SANS curves obtained at $47^{\circ} \mathrm{C}$ in polymer contrast from the $\mathrm{PEO}_{12}-b-\mathrm{PDMS}_{43}-b-\mathrm{PEO}_{12} / \mathrm{DPPC}-\mathrm{d}_{62}$ mixture at the lipid fraction $f_{d}=0.21$ (weight ratio), and best fit with the vesicle ('spherical shell') model (line)

To get more information regarding lateral organization of lipid and copolymer, Time Resolved Förster Energy Transfer experiments were performed. FRET efficiencies versus the molar $\%$ of acceptor were measured on vesicle suspensions and compared to predicted FRET values following experimental methodology and theoretical formalism described in ESI (section S 2.5) and in our previous study. ${ }^{15}$

The detection of FRET phenomenon between donor-labeled copolymer and acceptor-labeled lipid, confirms the presence of hybrid vesicles. (No energy transfer would occur in a mixture of pure polymersomes and liposomes). However, the recovered FRET values are below the theoretically determined values for the case of a homogeneous distribution of copolymers and DPPC for $21 \%$ and 15\% lipid (Figure 4 and S22 respectively).

In fact, FRET efficiencies fall within the infinite phase separation FRET limit for both compositions studied. This strongly suggests a significant heterogeneity in copolymer and lipid mixing. This drop in FRET efficiencies can only be rationalized by phase separation into structures of at least 25-50 nm size. Since SANS measurements did not detect domains within these dimensions, the measured FRET efficiencies must reflect the formation of pure liposomes along with hybrid vesicles, which results in a decrease of lipid acceptors in hybrid vesicles.

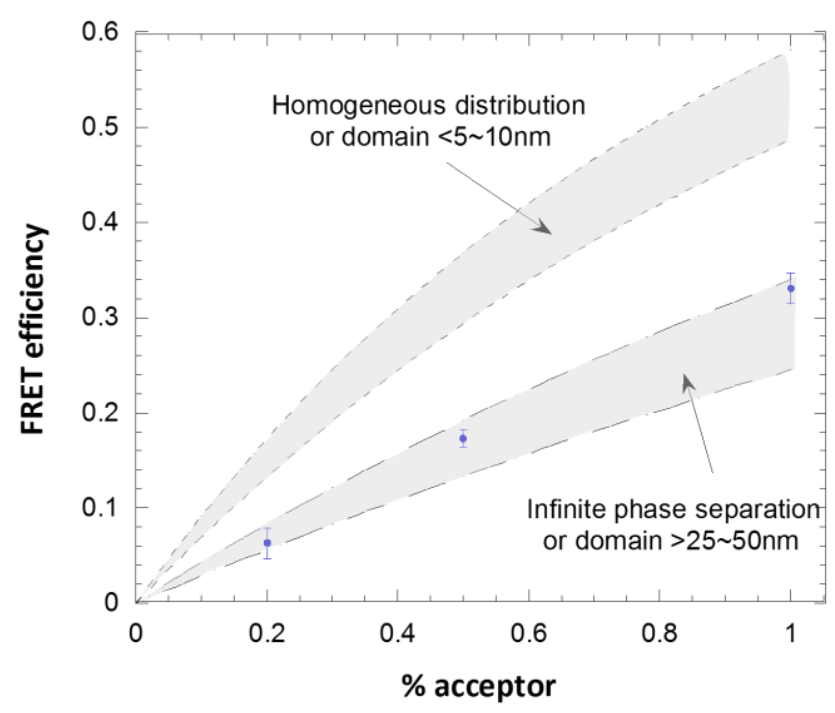

Figure 4: FRET efficiencies vs. content (mol \%) of lipidlabeled acceptor for $\mathrm{PEO}_{12}-b$-PDMS $43-b-\mathrm{PEO}_{12} / \mathrm{DPPC}(79 / 21$ $\mathrm{w} / \mathrm{w} \%$ ) mixtures at $46^{\circ} \mathrm{C}$. FRET efficiency values expected in case of homogenous distribution and infinite phase separation taking into account experimental uncertainty in area/polymer chain determination are delimited by shaded areas.

Cryo-TEM experiments performed on $\mathrm{PEO}_{12}-b$ $\mathrm{PDMS}_{43}-b-\mathrm{PEO}_{12} / \mathrm{DPPC}(79 / 21 \mathrm{w} / \mathrm{w} \%)$ reveal the presence of different types of vesicles : $25 \%$ are faceted and typical of pure DPPC vesicle, $65 \%$ are rounded vesicle, a few percentage is multilamellar or with a morphology in between rounded and faceted vesicle (R/F), with "smooth" angle. Faceted vesicles illustrate the presence of pure DPPC vesicles, and therefore uncomplete mixing of the components. Observations on a suspension frozen from $46^{\circ} \mathrm{C}$ revealed a strong increase of $\mathrm{R} / \mathrm{F}$ population accompanied by a strong decrease of faceted vesicles due to the typical behavior of pure DPPC becoming fluid at $46^{\circ} \mathrm{C}$. Complete statistics can be consulted in Supp. Info Fig S 38-S41. The strong increase at $46^{\circ} \mathrm{C}$ of the fraction of $\mathrm{R} / \mathrm{F}$ vesicles ( $\mathrm{ca}$. $37 \%$ ), which are morphologies observed classically neither for pure polymersomes nor pure DPPC vesicle, is interpreted as a convincing sign of the presence of hybrid polymersome-lipid vesicles. Additional signs of the presence of hybrid vesicles can be detected. Indeed the average membrane thickness distribution is shifted to slightly lower values compared to pure polymersomes $(7.3 \pm 1$ instead of $8.8 \pm 0.5 \mathrm{~nm}$ ). At $46^{\circ} \mathrm{C}$, another popula- 
tion appears at 5-6 nm (Fig S40) a membrane thickness typically observed for pure DPPC vesicles at such temperature by cryo-TEM.

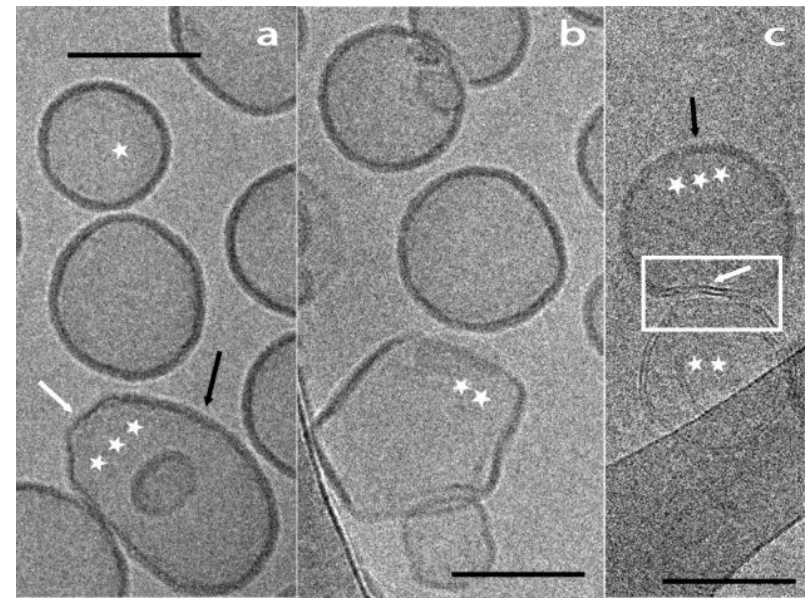

Figure 5. Cryo-TEM picture of 79/21 (weight ratio) PEO12-bPDMS43-b-PEO12 /DPPC mixture quenched from room temperature. The vesicle with one white star corresponds to a pure polymersome. The vesicle with two white stars represents a typical pure DPPC faceted vesicle (insert b). The vesicles with 3 white stars are hybrid vesicles, the white arrow points to the thinner membrane corresponding to the lipid part and the black arrow points to thicker membrane corresponding to the polymer part. The insert c illustrates nicely the Cryo-TEM signature of lipid domains (bilayer, pointed by the white arrow) in polymer membrane (pointed by the black arrow) obtained on a sample quenched from $46^{\circ} \mathrm{C}$. All scale bars represent $100 \mathrm{~nm}$.

This may result from the simple decrease of membrane thickness of DPPC vesicles expected when temperature increases, making them more visible compared to the polymer membrane thickness $(\sim 8$ $\mathrm{nm}$ ), or an eventual fission of pure DPPC membranes from hybrid vesicles. Again, it was possible to observe the cryo-TEM signature of a lipid domain in the surrounding polymer-rich membrane as illustrated in Figure 5. It is worth noticing that mixing DPPC with $\mathrm{PEO}_{12}-b-\mathrm{PDMS}_{43}-b-\mathrm{PEO}_{12}$ which is a copolymer forming vesicle with a membrane thickness around $8-9 \mathrm{~nm}$, results in a completely different behavior compared to $\mathrm{PEO}_{17}-b$ - $\mathrm{PDMS}_{67}-b$ $\mathrm{PEO}_{17} / \mathrm{DPPC}$ mixtures. In the case of $\mathrm{PEO}_{12}-b$ $\mathrm{PDMS}_{43}-b-\mathrm{PEO}_{12} / \mathrm{DPPC}$ mixtures, no worm-like hybrid nanostructures were obtained. Instead, copolymer chains and lipids self-assemble to form to some extent hybrid vesicles, whose exact membrane characteristics (presence of domains, size and number) cannot be quantified at this stage due to sample heterogeneity (coexistence of liposomes and hybrid vesicles with different lipid content). This reduces the potential of SANS and FRET analysis to recover quantitative information. We have therefore extended our investigations to block co- polymer $\mathrm{PEO}_{8}-b$ - $\mathrm{PDMS}_{22}-b-\mathrm{PEO}_{8}$, which form vesicles with membrane thickness close to those of liposomes $(\sim 5 \mathrm{~nm})$.

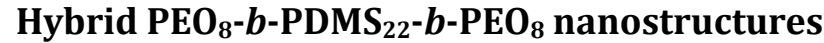

The new model of hybrid vesicles described at the end of the article was used to describe the $\mathrm{PEO}_{8}-b$ $\mathrm{PDMS}_{22}-b-\mathrm{PEO}_{8} / \mathrm{DPPC}-\mathrm{d}_{62}$ mixtures: the scattering curves obtained in polymer contrast were well fitted with a hybrid vesicle form factor. A typical fit is illustrated on Figure 6, and other fits can be consulted in supporting info. (Fig S22). Best fits parameters are reported in Table 3.

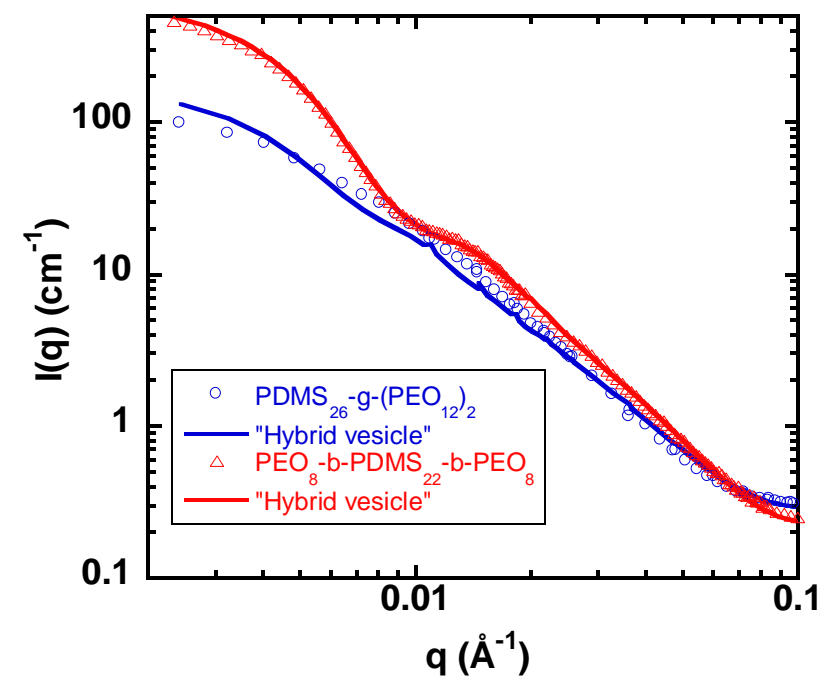

Figure 6. SANS curves obtained in polymer contrast at $20^{\circ} \mathrm{C}$ for mixtures at DPPC- $\mathrm{d}_{62}$ lipid fraction $f_{\mathrm{d}}=\mathbf{0 . 2 1}$ (weight ratio) for the two copolymers of nearly same molar mass and two different structures: $\mathrm{PEO}_{8}-b-\mathrm{PDMS}_{22}-b-\mathrm{PEO}_{8}$ and $\mathrm{PDMS}_{26}-g$ $\left(\mathrm{PEO}_{12}\right)_{2} \mathrm{r}$. Solid lines are best fits with the newly proposed 'hybrid vesicle' model.

The fits work reasonably well with about 8 to 12 disks of lipids of 2-4 nm diameters but with very high dispersity. Size of the vesicle obtained by the fitting procedure $\left(R_{\mathrm{v}}\right)$ is low, but with high dispersity as previously observed for $\mathrm{PEO}_{12}-b$ $\mathrm{PDMS}_{43}-b-\mathrm{PEO}_{12} / \mathrm{DPPC}$ mixtures. Thus the weight average radius taking into account $\sigma_{R_{\mathrm{V}}}$ becomes close to $R_{\mathrm{g}}$ (respectively $R_{\mathrm{w}}=31.5 \mathrm{~nm}$ at $20^{\circ} \mathrm{C}$ and $R_{\mathrm{w}}=36.2 \mathrm{~nm}$ at $47^{\circ} \mathrm{C}$ ). However, such high dispersity does not allow detection of a possible effect of temperature or lipid fraction on the evolution of the number $\left(N_{\mathrm{d}}\right)$ or of the size $\left(R_{\mathrm{d}}\right)$ of the lipid domains.

In lipid contrast, the scattering curves can be reasonably fitted with a vesicle form factor, (Fig S 23) and the corresponding fit parameters are indicated in Table 3. Again, vesicle radii are rather low with high dispersity, but as previously mentioned in 
good agreement with the radii of gyration obtained dispersity $\left(\sigma_{\mathrm{R}_{\mathrm{v}}}\right)$. by a Guinier plot, when taking into account size

\begin{tabular}{|c|c|c|c|c|c|c|c|c|c|c|c|c|c|c|}
\hline & \multicolumn{8}{|c|}{ Polymer contrast $\left(P E O_{8}-b-P D M S_{22}-b-P E O_{8}\right)$} & \multicolumn{5}{|c|}{ Lipid contrast } \\
\hline & & \multirow{2}{*}{$\begin{array}{l}\text { Guinier } \\
R_{g}(n m)\end{array}$} & \multicolumn{7}{|c|}{ Hybrid vesicle model } & \multirow{2}{*}{$\begin{array}{c}\text { Guinier } \\
R_{g} \\
(\mathrm{~nm})\end{array}$} & \multicolumn{4}{|c|}{ Vesicle model } \\
\hline$f_{d}$ & $T$ & & $R_{v}(\mathrm{~nm})$ & $\sigma_{R_{v}} *$ & $N_{d}$ & $\begin{array}{c}R_{d} \\
(\mathrm{~nm})\end{array}$ & $\sigma_{R d}$ & $\begin{array}{l}\delta_{v}{ }^{*} \\
(\mathrm{~nm})\end{array}$ & $\begin{array}{c}\delta_{d}{ }^{*} \\
(\mathrm{~nm})\end{array}$ & & $\begin{array}{l}R_{v} \\
(\mathrm{~nm})\end{array}$ & $\sigma_{R v}$ & $\begin{array}{c}\delta_{v} \\
(\mathrm{~nm})\end{array}$ & $\sigma_{\delta v}$ \\
\hline \multirow{2}{*}{0.21} & $20^{\circ} \mathrm{C}$ & $33 \cdot 5$ & 22 & 0.32 & $7 \cdot 9$ & 1.9 & 0.9 & $5 \cdot 5$ & $4 \cdot 3$ & $33 \cdot 3$ & 16 & 0.4 & 3.2 & 0.19 \\
\hline & $47^{\circ} \mathrm{C}$ & 39 & $25 \cdot 3$ & 0.32 & 12 & 1.4 & 0.38 & $5 \cdot 5$ & 3.2 & 41.6 & 28 & $0.33^{*}$ & 3.0 & 0.11 \\
\hline & & \multicolumn{8}{|c|}{ Polymer contrast $P D M S_{26}-g-\left(P E O_{12}\right)_{2}$} & \multicolumn{5}{|c|}{ Lipid contrast } \\
\hline \multirow{2}{*}{0.15} & $20^{\circ} \mathrm{C}$ & 32.5 & 8.5 & 1.2 & $5 \cdot 7$ & 2.9 & 3.12 & 5.6 & $4 \cdot 3$ & $33 \cdot 5$ & \multirow{4}{*}{\multicolumn{4}{|c|}{ Shell fit is not possible }} \\
\hline & $47^{\circ} \mathrm{C}$ & 32.2 & 11.2 & 1.2 & 4 & 4.2 & 3.12 & 5.6 & 3.2 & 41.0 & & & & \\
\hline \multirow{2}{*}{0.21} & $20^{\circ} \mathrm{C}$ & 31.8 & 11.7 & 1.2 & $3 \cdot 7$ & 7.2 & 1.25 & 5.6 & $4 \cdot 3$ & 34.0 & & & & \\
\hline & $47^{\circ} \mathrm{C}$ & 31.9 & 14.7 & 1.2 & 5.8 & $3 \cdot 3$ & 3 & 5.6 & 3.2 & 40.0 & & & & \\
\hline
\end{tabular}

Table 3. Best fit parameters obtained with 'holey shell' form factor (or 'hybrid vesicle' model) in polymer contrast and vesicle ('spherical shell') form factor in lipid contrast. *: fixed parameters. $R_{\mathrm{v}}$ : radius of vesicle. $\sigma_{R v}$ : log-normal dispersion width. $N_{\mathrm{d}}$ : number of disk-shaped holes (lipid domains). $R_{\mathrm{d}}$ : radius of the holes. $\boldsymbol{\delta}_{\mathbf{v}}:$ membrane thickness of vesicle. $\boldsymbol{\delta}_{\mathbf{d}}:$ membrane thickness of lipid domains.

Lipid membrane thickness is however determined with a better precision $(\sigma=0.1 \sim 0.2)$ and seems lower compared to values obtained for pure lipid vesicles $\left(4.3 / 3.1 \mathrm{~nm}\right.$ at $25^{\circ} \mathrm{C} / 47^{\circ} \mathrm{C}$ ) (See Table S8). This could be a sign of better dispersion of lipids in polymer membranes and a lower number of pure liposomes compared to the $\mathrm{PEO}_{12}-b-\mathrm{PDMS}_{43}-b$ $\mathrm{PEO}_{12}$ /DPPC mixtures.

Cryo-TEM images again reveal three different morphologies: rounded, rounded and faceted $(\mathrm{R} / \mathrm{F})$, and faceted vesicles, which represent respectively $\sim 21 \%, 23 \%$ and $56 \%$ of the population. Rounded vesicles are likely typical of polymersomes either pure or in which a low amount of DPPC is dispersed, while faceted vesicles are typical of DPPC-rich vesicles. Complete statistics can be consulted in supporting information (Fig S42-S45). Regarding membrane thickness, at $20^{\circ} \mathrm{C}$ it seems that two populations appear (Fig S44). One is centered between 5 and $5.5 \mathrm{~nm}$, which corresponds to the membrane thickness of pure $\mathrm{PEO}_{8}-b$ - $\mathrm{PDMS}_{22}-b-\mathrm{PEO}_{8}$ vesicles. Another one is between 7 and $7.5 \mathrm{~nm}$, closer to the $6.2 \pm 0.4 \mathrm{~nm}$ apparent thickness observed for pure DPPC vesicles by Cryo-TEM (See Supp. Info of Dao et al. ${ }^{15}$ and Table 1). However, increasing temperature results into a decrease of the faceted vesicle population but also of the R/F vesicle fraction, accompanied by an increase of the population of rounded vesicles. Interestingly, the membrane thickness values of the population of vesicles seem to get more uniform around a similar average value, with a strong decrease of the population of vesicles with membrane thickness between 5 and $5.5 \mathrm{~nm}$. As the membrane thickness of pure polymersomes does not change with temperature, this is again a good indication of the presence of hybrid vesicles.

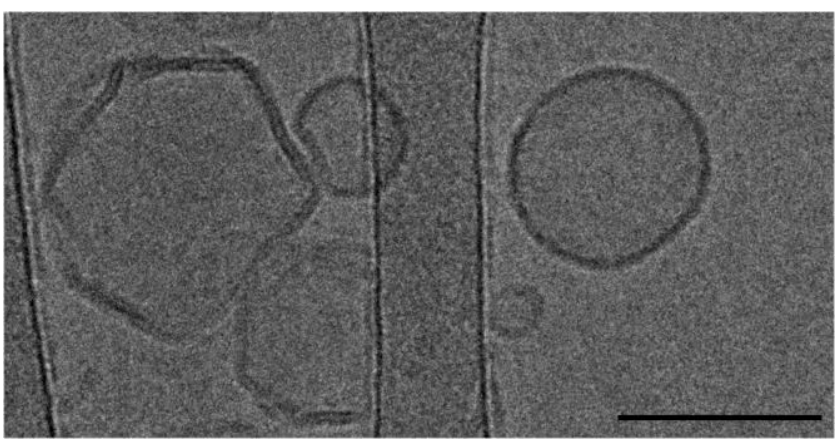

Figure 7. Cryo-TEM picture of 79/21 weight ratio $\mathrm{PEO}_{8}-b$ $\mathrm{PDMS}_{22}-b-\mathrm{PEO}_{8} / \mathrm{DPPC}$ mixture quenched from room temperature. Scale bar length is $100 \mathrm{~nm}$.

Additional aid from the fluorescence spectroscopy can bring more evidences on the hybrid nature of the membranes. The determined non-zero values of FRET efficiency again confirm the presence of hybrid vesicles to a given extent. The values are still in the infinite phase separation limit for $21 \%$ and 15\% lipid (Figure 8 and S23 respectively). Therefore, considering SANS, Cryo-TEM and FRET, it seems reasonable to conclude that as for the previous copolymer $\mathrm{PEO}_{12}-b$ - $\mathrm{PDMS}_{43}-b-\mathrm{PEO}_{12}$, a significant part of the lipid is dispersed in the polymer phase, leading to the formation of hybrid vesicles. On the other hand, as observed for $\mathrm{PEO}_{12}$ $b$-PDMS $43-b$ - $\mathrm{PEO}_{12}$, pure liposomes are also 
formed, and therefore lead to a global decrease of the FRET efficiency. It is however difficult to estimate the relative amount of hybrid vesicles in the $\mathrm{PEO}_{12}-b-\mathrm{PDMS}_{43}-b-\mathrm{PEO}_{12} / \mathrm{DPPC}$ and $\mathrm{PEO}_{8}-b-$ $\mathrm{PDMS}_{22}-b-\mathrm{PEO}_{8} / \mathrm{DPPC}$ mixtures. However, in the case of $\mathrm{PEO}_{8}-b$ - $\mathrm{PDMS}_{22}-b-\mathrm{PEO}_{8}$, the homogenization of membrane thickness with temperature and the good quality of the fit obtained using the "hybrid vesicle' form factor in polymer contrast to fit the SANS data, suggest that pure liposomes and polymersomes are less numerous, as ascribed from a lower hydrophobic mismatch than with the larger molar mass triblock copolymer.

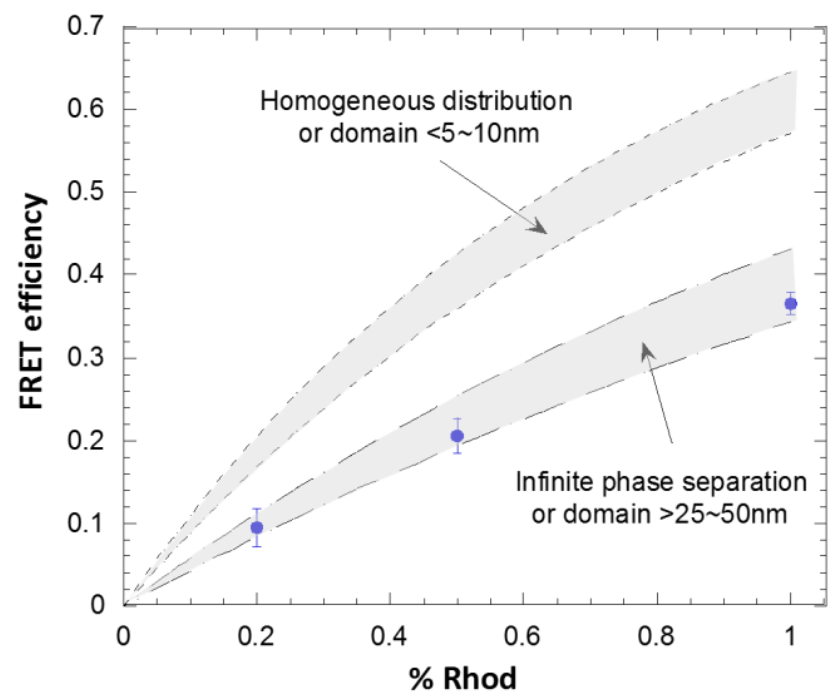

Figure 8. FRET efficiencies vs. content (mol \%) of lipidlabeled acceptor for $\mathrm{PEO}_{8}-b-\mathrm{PDMS}_{22}-b-\mathrm{PEO}_{8} / \mathrm{DPPC}(79 / 21$ $\mathrm{w} / \mathrm{w} \%)$ mixtures at $46{ }^{\circ} \mathrm{C}$. Theoretical expectation for the case of homogenous distribution and infinite phase separation taking into account experimental uncertainty in the area/polymer chain determination are delimited by shaded areas.

\section{Hybrid PDMS ${ }_{26}-g-\left(\mathrm{PEO}_{12}\right)_{2}$ nanostructures}

As the use of the triblock copolymer based on PDMS presenting membrane thickness of $5 \mathrm{~nm}$ close to those of liposomes did not lead to the formation of a large majority of LHUVs with DPPC, we extended our studies to the association of a grafted copolymer, $\mathrm{PDMS}_{26}-g$ - $\left(\mathrm{PEO}_{12}\right)_{2}$ with DPPC. This is a system that we have previously studied and for which a phase separation inside the membrane has been shown especially with the use of a basic flat disk form factor to fit the SANS data in different matching conditions. ${ }^{13}$ This system is particularly interesting as it allows the evaluation of an architecture effect (graft vs. triblock), the membrane thickness and chemical nature of the block being identical to $\mathrm{PEO}_{8}-b-\mathrm{PDMS}_{22}-b-\mathrm{PEO}_{8}$. Two lipid fractions $f_{\mathrm{d}}$ were studied, $15 \%$ and $21 \% \mathrm{w} / \mathrm{w}$. In this system, the whole vesicular structures are very polydisperse and almost no oscillation is visible on the SANS curves, unlike what was observed with $\mathrm{PEO}_{8}-b$-PDMS22- $b$ - $\mathrm{PEO}_{8} /$ DPPC mixtures. In polymer contrast, since changes upon temperature variation were observed in the same intermediate $q$ range as for the previous triblock copolymer, the scattered intensity was also fitted with the "hybrid vesicle' model described in the last section of the article. Typical fits are shown in Figure 6 and the best fit parameters reported in Table 3.

Despite the high polydispersity, the curves could be reasonably fitted with the 'hybrid vesicle' form factor. Although the values obtained for the radius of the vesicles are meaningless, the fit accounted well any variation in the scattering curves upon temperature changes. On average, whatever the temperature or lipid fraction, we find about 4-6 disks of 6-14 nm diameter, very polydisperse in size. This suggests the formation of small domains of lipid in the polymer membrane. Interestingly, in lipid contrast, as for the previous triblock copolymer, data cannot be fitted with the simple vesicle ('spherical shell') form factor model, suggesting again that lipids are not simply homogenously distributed within the polymer membrane.

To get more insight into these $\mathrm{PDMS}_{26}-g$ $\left(\mathrm{PEO}_{12}\right)_{2} / \mathrm{DPPC}$ nanostructures, FRET measurements were also performed and compared to the prediction of homogenous distribution of donor and acceptor probes within the membranes. In this case, experimental points fall in the range predicted for domains size below or close to the Förster radius of the donor-acceptor FRET pair considered here $(5 \mathrm{~nm})$.

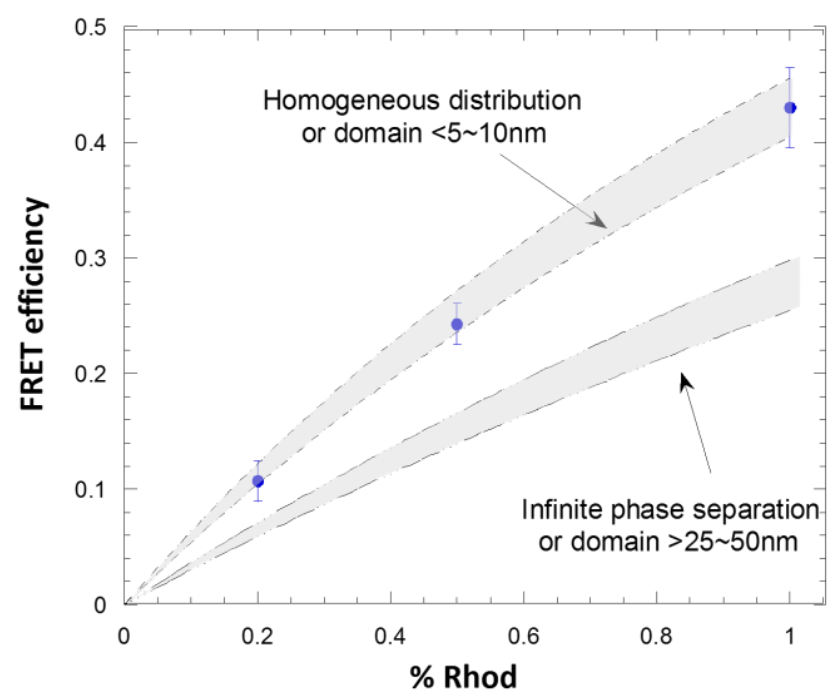

Figure 9. FRET efficiencies vs. \% molar of acceptor at $50^{\circ} \mathrm{C}$ in 
mixture $\mathrm{PDMS}_{26}-\mathrm{g}-\left(\mathrm{PEO}_{12}\right)_{2} / \mathrm{DPPC}$ with $f_{\mathrm{d}}=0.15$ mass of DPPC. Theoretical expectations for the cases of homogenous distribution and infinite phase separation respectively, taking into account the experimental uncertainty in area/polymer chain determination by SLS and Langmuir compression isotherms, as delimited by shaded areas.

Additionally, their presence with dimensions suggested by SANS would remain undetected by the FRET measurements. In this way, although a comparison of recovered domain sizes is not possible between FRET and SANS analyses due to the limitations in interpreting FRET efficiencies, the presence of nanoscale lipid domains in hybrid $\mathrm{PDMS}_{26}$ $g$ - $\left(\mathrm{PEO}_{12}\right)_{2} / \mathrm{DPPC}$ vesicles with dimensions suggested from SANS results $(6-14 \mathrm{~nm})$ is fully in line with the results obtained by FRET measurements. In such systems, the association of the copolymer and lipid molecules within the same membrane is more efficient and the number of pure polymersomes and liposomes which inevitably perturb the SANS analysis and decreases the FRET efficiency, is lower (although still present, as illustrated by Cryo-TEM (Fig S46-S50)) than for the previously considered copolymers, rendering the "hybrid vesicle" form factor more pertinent to get information about the membrane structure of these hybrid vesicles.

\section{CONCLUSIONS}

The film rehydration and extrusion technique for the production of hybrid polymer/lipid large unilamellar hybrid vesicles (LHUVs) appears as a non-ideal procedure, although commonly used in the literature.5-7, 9, 26 Marrying of a single membrane triblock or diblock copolymer having high molar mass with phospholipids has already been achieved for GHUVs, although it has been reported that some mixtures did not give any vesicles ${ }^{14}$ or led to the budding or eventual fission into separated liposomes and polymersomes. ${ }^{27}$ In the case of LHUVs, the association seems driven by more subtle features. It is believed that a high line tension resulting from the high differences in membrane thickness would lead to formation of separated liposomes and polymersomes, but other hybrid structures can be formed as illustrated by coreshell tubular (worm-like) or 'pan-like' micelles obtained with $\mathrm{PEO}_{17}-b$-PDMS $67-b$ - $\mathrm{PEO}_{17} / \mathrm{DPPC}$ mixtures. Decreasing the molar mass of triblock copolymer results into the disappearance of these hybrid 'worm-like' or 'pan-like' micelles and to the formation, at least to some extent, of hybrid vesicles. Their membrane structure cannot be characterized precisely because of the additional pres- ence of pure liposomes and polymersomes. This heterogeneity seems to decrease when the molar mass of copolymer further decreases, leading to the formation of a more homogeneous vesicle population with membrane thicknesses close to that of liposomes $(\sim 5 \mathrm{~nm})$ however the values obtained with 'hybrid vesicle' form factor in the case of $\mathrm{PEO}_{8}-b-\mathrm{PDMS}_{22}-b-\mathrm{PEO}_{8} / \mathrm{DPPC}$ mixtures in term of number and size of lipid domains in a vesicle should not be considered as precise values, as the scattering coming from hybrid vesicles is somewhat screened by scattering of pure polymersomes. Interestingly, changing the copolymer architecture from triblock to grafted while maintaining the chemical nature, molar mass and membrane thickness unchanged, leads to a considerably more efficient mixing of the lipid and polymer molecules into the same membrane, generating hybrid vesicles presenting only a few lipid nanodomains of several nanometers $(3 \sim 7 \mathrm{~nm}$ radius). This indicates that line tension at the lipid/polymer boundaries can be modulated to some extend through the architecture of the copolymer, in addition to the molar mass and chemical nature of the block. Although it is premature to generalize our results to all kinds of copolymer/lipid assemblies, it is important to realize that association of copolymers and lipids that could be obtained on giant unilamellar hybrid vesicles (GHUVs) does not systematically reflect what happens at the nanoscale. In the case of LHUVs, higher curvature energy may add to the hydrophobic mismatch and chemical incompatibility between lipid tails and hydrophobic copolymer blocks. Therefore, a great attention has to be paid on the characterization of such structures before going further in the evaluation of their physical (e.g. membrane permeability, drug release, phase behavior) or biofunctional properties (e.g. protein insertion, biorecognition) properties. Techniques available in-lab such as Dynamic Light Scattering and Cryo-TEM commonly used to characterize classical LUVs may be insufficient to confirm the hybrid character of the vesicles. In addition, counting techniques such as Flow cytometry suffer from a lack of sensitivity at such vesicle size $(100 \mathrm{~nm})$. Beyond the issue of solving properly the hybrid character and membrane structure of such assemblies, it appears essential to think about other methods to marry block copolymers with high molecular weight and phospholipids into LHUVs in an efficient way, by playing either on molecular 
aspects (e.g. cholesterol addition ${ }^{5}$ ) or by introducing new formulation processes.

\section{ANNEX: 'Hybrid vesicle' form Factor}

While various form factors of core-shell particles, multiple layers or single layer vesicles have been reviewed $^{28}$ and corresponding fitting routines available (SasView, http://www.sasview.org $/$ ), none of these scattering functions are suitable for (polymer) vesicles with (lipid) patches. Nonetheless, a model of scattering by lipid vesicles with lateral heterogeneities in the membrane has been recently developed by using Monte Carlo modeling with a modified coarse-graining method ${ }^{29}$. This structural model approach is very powerful since it provides important qualitative information about lipid mixing. However, it is computationally expensive and time limiting since it does not lead to an analytical form factor for multi-domain vesicles. Therefore, in order to overcome this lack of analytical expressions, the same group utilized a spherical harmonic expansion of the vesicle form factor to obtain an analytical solution for the scattering from vesicles containing a single round domain ${ }^{30}$. Very recently, they extended their model with a general theory for scattering from laterally heterogeneous vesicles of arbitrary size and spatial configuration ${ }^{31}$. They obtained analytical expressions of the form factor and scattering intensity that they compared to corresponding Monte-Carlo calculations. While this approach is very promising, these expressions are not enough simplified, and together with problems of sizes distributions and fitting routines methods to implement, it was not feasible to use them to fit our SANS data.

Instead, we have developed a more simple approach to try to describe the scattering of hybrid vesicles of polymers with lipid domains, using only classical expressions of scattering amplitudes and form factors that can be easily processed with proven fitting methods. We derived a model recently proposed by Bergström et al.32 to describe mixtures of surfactants bilayers with salt creating holes inside the bilayers. In this simplified model of perforated vesicles, several assumptions were made:

i) the vesicles were considered as infinitely thin circular shells with radius $R$, of form factor:

$$
P_{\mathrm{v}}(q, R)=\left[F_{\mathrm{v}}(q, R)\right]^{2}=\left[\frac{\sin q R}{q R}\right]^{2} \quad \mathrm{Eq} \mathrm{A}_{1}
$$

the holes being considered as symmetrical disks with radius $R_{\mathrm{h}}$ and amplitude:

$$
F_{\mathrm{h}}=\left(\frac{2\left[1-B_{1}\left(2 q R_{\mathrm{h}}\right) / q R_{\mathrm{h}}\right]}{q R_{\mathrm{h}}}\right)^{1 / 2} \quad \text { Eq. A } 2
$$

where $B_{1}(\mathrm{x})$ is the Bessel function of the first order.

The form factor of perforated bilayer vesicles was obtained by subtracting the contribution from the holes to the one of a bilayer vesicle with an appropriate weighting of the areas (equivalent to volume for infinitely thin vesicles), i.e.:

$$
S_{\text {per }}=\left(\frac{A_{\text {bil }} F_{\text {bil }}-N_{\mathrm{h}} A_{\mathrm{h}} F_{\mathrm{h}}}{A_{\mathrm{bil}}-N_{\mathrm{h}} A_{\mathrm{h}}}\right)^{2} \quad \text { Eq. A } 3
$$

where $A_{\mathrm{h}}=\pi \cdot R_{\mathrm{h}}^{2}$ is the area per hole, $N_{\mathrm{h}}$ the number of holes and $A_{\mathrm{bil}}=4 \pi \cdot R_{\mathrm{v}}^{2}$ the area of the infinitely thin bilayer vesicle.

Hybrid vesicles are slightly different from perforated vesicles since the holes filled with solvent in the vesicles are replaced by disk-like domains filled with lipids. In addition, we also wished to account for the different thicknesses of the polymer and of the lipid membranes. So, we considered a model of hybrid vesicles composed of a bilayer polymer vesicle with bilayer 'disks' of lipids as shown on Figure 10: a vesicle with inner radius $R_{\mathrm{v}}$ and thickness $\delta_{\mathrm{v}}$, containing $N_{\mathrm{d}}$ disks of us $R_{\mathrm{d}}$ and membrane thickness $\delta_{\mathrm{d}}$.

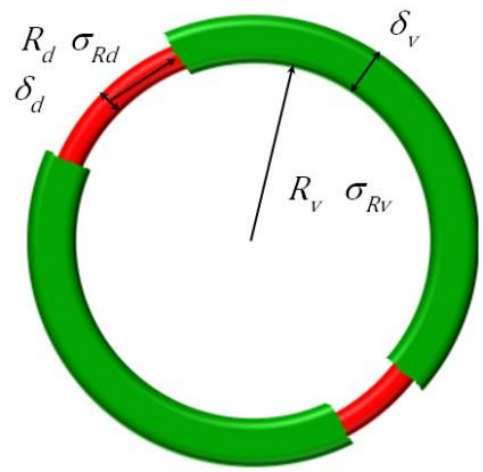

Figure 10: Scheme of the 'hybrid vesicle' model: a vesicle with inner radius $R_{\mathrm{v}}$ (distribution $\sigma_{R_{\mathrm{v}}}$ ) and thickness $\delta_{\mathrm{v}}$, containing $N_{\mathrm{d}}$ disks of radius $R_{\mathrm{d}}$ (distribution $\sigma_{R_{\mathrm{d}}}$ ) and thickness $\delta_{\mathrm{d}}$.

In order to account for the respective disk/vesicle thicknesses, we introduced the scattering amplitude of a bilayer cross-section ${ }^{32}$ given by:

$$
F_{b, \xi}=\frac{\sin q \xi}{q \xi} \quad \text { Eq. A } 4
$$


where $\xi$ is half the bilayer thickness $\left(\xi=\frac{\delta}{2}\right)$, and in our case, of either the polymer or of the lipid membrane.

The analytical expression of the form factor employed is indicated in Eq. A5, where $\rho_{\mathrm{v}}, \rho_{\mathrm{d}}, \rho_{\mathrm{o}}$ are the neutron scattering length densities of the vesicle (polymer), of disks (lipid) and of the solvent. $\delta_{\mathrm{v}}$, and $\delta_{\mathrm{d}}$ correspond respectively to the membrane thickness of polymer vesicle and of the lipid disks. $F_{\mathrm{v}}\left(q, R_{\mathrm{v}}+\delta_{\mathrm{v}} / 2\right)$ is the scattering amplitude of an infinitely thin circular shell with radius $R_{\mathrm{v}}+\frac{\delta_{\mathrm{v}}}{2}$ (Eq. A1). $F_{\mathrm{h}, R_{\mathrm{d}}}$ is the scattering amplitude of symmetrical circles with radius $R_{\mathrm{d}}$ (Eq. A2) $F_{\mathrm{b}, \delta_{\mathrm{v}}}$ (respectively $\left.F_{\mathrm{b}, \delta_{\mathrm{d}}}\right)$ is the scattering amplitude of a bilayer cross-section of thickness $\delta_{\mathrm{v}}$ (respectively $\delta_{\mathrm{d}}$ ) (Eq. A4). $V_{\mathrm{d}}$ is the volume of one disk geometrically approximated by $V_{\mathrm{d}}=\pi R_{\mathrm{d}}^{2} \delta_{\mathrm{d}}$, and $V_{\mathrm{v}}$ is the volume of a vesicle membrane geometrically approximated by $V_{\mathrm{v}}=4 \pi\left(R_{\mathrm{v}}+\delta_{\mathrm{v}} / 2\right)^{2} \delta_{\mathrm{v}}$.

As in Bergström et al. ${ }^{32}$ we subtracted the scattering amplitude of $N_{\mathrm{d}}$ disks of thickness $\delta_{\mathrm{v}}$, but in order to account for the scattering of full disks instead of holes, we have added a contribution of disks of thickness $\delta_{\mathrm{d}}$, with their own scattering length density $\rho_{\mathrm{d}}$. For all these contributions, the thicknesses have been taken into account according to Eq. A4. Moreover, the scattering amplitudes of the different contributions were weighted by the corresponding volumes, instead of the areas in the work of Bergström et al. ${ }^{32}$

As a result, the 'hybrid vesicle' model has several parameters: $R_{\mathrm{v}}, \delta_{\mathrm{v}}, R_{\mathrm{d}}, \delta_{\mathrm{d}}$, and possibly a (Lognormal) distribution for each of these parameters, as well as the number of disks $N_{\mathrm{d}}$. The scattering length densities $\rho_{\mathrm{v}}, \rho_{\mathrm{d}}$ can also be different from the ones of the pure compounds if we assume that a phase separation between lipids and polymers occurs within the vesicle membrane. Finally, for polydisperse hybrid vesicles, this model has $11 \mathrm{fit}$ parameters. Considering our data, up to a $q$ value about $0.12 \AA^{-1}$ where the scattering generally reaches the incoherent background, the number of data points $N \approx 150$. Therefore, in order to reasonably describe the main tendencies governing the phase separation occurring in our systems, we reduced the number of fit parameters by using the following assumptions: the scattering length densities are those of pure compounds, the bilayer thickness of polymers and of lipids $\left(\delta_{\mathrm{v}}, \delta_{\mathrm{d}}\right)$ are fixed to the values measured for the pure compound vesicles. No size distribution is used for these two parameters since the fitted distributions obtained on pure compounds are weak (0.12-0.18 assuming a log-normal distribution) and accounting for the $q$ resolution of SANS experiments, they will affect the scattering curves at very large $q$ values, not very informative here.

Thus 5 parameters only allow us to describe the phase separation occurring in the hybrid vesicles: those are $R_{\mathrm{v}}, \sigma_{R_{\mathrm{v}}}, N_{\mathrm{d}}, R_{\mathrm{d}}, \sigma_{R_{\mathrm{d}}}$. Data fitting was performed by writing a Matlab ${ }^{\circledR}$ function according to Eq A5. and using Matlab ${ }^{\circledR}$ methods for nonlinear least square curve-fit. Throughout all the fits with Matlab $^{\circledR}$ and SasView programs, corrections were made for instrumental smearing. 33-34

$$
\begin{aligned}
& S_{\text {hybrid vesicle }} \\
& =\left(\frac{\left(\rho_{\mathrm{v}}-\rho_{\mathrm{o}}\right) V_{\mathrm{v}} F_{\mathrm{b}, \delta_{\mathrm{v}}} F_{\mathrm{v}}\left(q, R_{\mathrm{v}}+\delta_{\mathrm{v}} / 2\right)-\left(\rho_{\mathrm{v}}-\rho_{\mathrm{o}}\right) N_{\mathrm{d}} \pi R_{\mathrm{d}}^{2} \delta_{\mathrm{v}} F_{\mathrm{b}, \delta_{\mathrm{v}}} F_{\mathrm{h}, R_{\mathrm{d}}}+\left(\rho_{\mathrm{d}}-\rho_{\mathrm{o}}\right) N_{\mathrm{d}} V_{\mathrm{d}} F_{\mathrm{b}, \delta_{\mathrm{d}}} F_{\mathrm{h}, R_{\mathrm{d}}}}{V_{\mathrm{v}}-N_{\mathrm{d}}\left(\pi R_{\mathrm{d}}^{2} \delta_{\mathrm{d}}\right)+N_{\mathrm{d}} V_{\mathrm{d}}}\right)^{2}
\end{aligned}
$$

Eq. $A_{5}$ 


\section{FUNDING SOURCES}

International doctoral school on Functional Materials (IDS-FunMat), Erasmus Mundus (EU), FCT (RECI/CTM-POL/0342/2012,

FAPESP/20107/2014, UID/NAN/50024/2013) Agence Nationale de la Recherche (KbT 12-BS080018-01)

\section{ACKNOWLEDGMENT}

This work benefitted from use of the SasView software, originally developed by the DANSE project under NSF award DMR-0520547

\section{REFERENCES}

1. Le Meins, J. F.; Schatz, C.; Lecommandoux, S.; Sandre, O., Hybrid polymer/lipid vesicles: state of the art and future perspectives. Materials Today 2013, 16 (10), 397-402.

2. Schulz, M.; Binder, W. H., Mixed Hybrid Lipid/Polymer Vesicles as a Novel Membrane Platform. Macromol Rapid Commun 2015, 36 (23), 2031-41.

3. Morimoto, N.; Sasaki, Y.; Mitsunushi, K.; Korchagina, E.; Wazawa, T.; Qiu, X.-P.; Nomura, S.-i. M.; Suzuki, M.; Winnik, F. M., Temperatureresponsive telechelic dipalmitoylglyceryl poly(Nisopropylacrylamide) vesicles: real-time morphology observation in aqueous suspension and in the presence of giant liposomes. Chem. Commun. 2014, 50 (61), 8350-8352.

4. Henderson, I. M.; Paxton, W. F., Salt, Shake, Fuse-Giant Hybrid Polymer/Lipid Vesicles through Mechanically Activated Fusion. Angewandte Chemie-International Edition 2014, 53 (13), 33723376.

5. Winzen, S.; Bernhardt, M.; Schaeffel, D.; Koch, A.; Kappl, M.; Koynov, K.; Landfester, K.; Kroeger, A., Submicron hybrid vesicles consisting of polymer-lipid and polymer-cholesterol blends. Soft Matter 2013, 9 (25), 5883-5890.

6. Lim, S.; de Hoog, H.-P.; Parikh, A.; Nallani, M.; Liedberg, B., Hybrid, Nanoscale Phospholipid/Block Copolymer Vesicles. Polymers 2013, 5 (3), 1102-1114.

7. Cheng, Z.; Elias, D. R.; Kamat, N. P.; Johnston, E.; Poloukhtine, A. A.; Popik, V. V.; Hammer, D. A.; Tsourkas, A., Improved Tumor Targeting of Polymer-based Nanovesicles Using Polymer-Lipid Blends. Bioconjugate Chem. 2011, 22 (10), 2021-2029.

8. Shen, W.; Hu, J.; Hu, X., Impact of amphiphilic triblock copolymers on stability and permeability of phospholipid/polymer hybrid vesicles. Chem. Phys. Lett. 2014, 600, 56-61.

9. Pippa, N.; Kaditi, E.; Pispas, S.; Demetzos, C., PEO-b-PCL-DPPC chimeric nanocarriers: selfassembly aspects in aqueous and biological media and drug incorporation. Soft Matter 2013, 9 (15), 4073-4082.

10. Bixner, O.; Bello, G.; Virk, M.; Kurzhals, S.; Scheberl, A.; Gal, N.; Matysik, A.; Kraut, R.; Reimhult, E., Magneto-Thermal Release from Nanoscale Unilamellar Hybrid Vesicles. ChemNanoMat 2016, 10.1002/cnma.201600278.

11. Su, X.; Mohamed Moinuddeen, S. K.; Mori, L.; Nallani, M., Hybrid polymersomes: facile manipulation of vesicular surfaces for enhancing cellular interaction. Journal of Materials Chemistry $B$ 2013, 1 (42), 5751-5755.

12. Pippa, N.; Stellas, D.; Skandalis, A.; Pispas, S.; Demetzos, C.; Libera, M.; Marcinkowski, A.; Trzebicka, B., Chimeric lipid/block copolymer nanovesicles: Physico-chemical and biocompatibility evaluation. European Journal of Pharmaceutics and Biopharmaceutics 2016, 107, 295-309.

13. Chemin, M.; Brun, P. M.; Lecommandoux, S.; Sandre, O.; Le Meins, J. F., Hybrid polymer/lipid vesicles: fine control of the lipid and polymer distribution in the binary membrane. Soft Matter 2012, 8, 2867-2874.

14. Nam, J.; Beales, P. A.; Vanderlick, T. K., Giant Phospholipid/Block Copolymer Hybrid Vesicles: Mixing Behavior and Domain Formation. Langmuir 2011, 27 (1), 1-6.

15. Dao, T. P. T.; Fernandes, F.; Er-Rafik, M.; Salva, R.; Schmutz, M.; Brulet, A.; Prieto, M.; Sandre, 0.; Le Meins, J. F., Phase Separation and Nanodomain Formation in Hybrid Polymer/Lipid Vesicles. ACS Macro Letters 2015, 4, 182-186.

16. Heberle, F. A.; Petruzielo, R. S.; Pan, J.; Drazba, P.; Kucerka, N.; Standaert, R. F.; Feigenson, G. W.; Katsaras, J., Bilayer Thickness Mismatch Controls Domain Size in Model Membranes. J. Am. Chem. Soc. 2013, 135 (18), 6853-6859.

17. Stefl, M.; Sachl, R.; Humpolickova, J.; Cebecauer, M.; Machan, R.; Kolarova, M.; Johansson, L. B. A.; Hof, M., Dynamics and Size of CrossLinking-Induced Lipid Nanodomains in Model Membranes. Biophys. J. 2012, 102 (9), 2104-2113.

18. Sachl, R.; Humpolickova, J.; Stefl, M.; Johansson, L. B.; Hof, M., Limitations of electronic energy transfer in the determination of lipid nanodomain sizes. Biophys J 2011, 101 (11), L60-2. 19. de Almeida, R. F.; Loura, L. M.; Fedorov, A.; Prieto, M., Lipid rafts have different sizes 
depending on membrane composition: a timeresolved fluorescence resonance energy transfer study. J Mol Biol 2005, 346 (4), 1109-20.

20. Suga, K.; Umakoshi, H., Detection of Nanosized Ordered Domains in DOPC/DPPC and DOPC/Ch Binary Lipid Mixture Systems of Large Unilamellar Vesicles Using a TEMPO Quenching Method. Langmuir 2013, 29 (15), 4830-4838.

21. Itel, F.; Chami, M.; Najer, A.; Loercher, S.; Wu, D.; Dinu, I. A.; Meier, W., Molecular Organization and Dynamics in Polymersome Membranes: A Lateral Diffusion Study. Macromolecules 2014, 47 (21), 7588-7596.

22. Nagle, J. F.; Tristram-Nagle, S., Structure of lipid bilayers. Biochimica et Biophysica Acta (BBA) Reviews on Biomembranes 2000, 1469 (3), 159195.

23. Nagle, J. F.; Wiener, M. C., Structure of fully hydrated bilayer dispersions. Biochimica et Biophysica Acta (BBA) - Biomembranes 1988, 942 (1), 1-10.

24. Safran, S. A.; Pincus, P. A.; Andelman, D.; MacKintosh, F. C., Stability and phase behavior of mixed surfactant vesicles. Phys Rev A 1991, 43 (2), 1071-1078.

25. Khan, S.; Li, M.; Muench, S. P.; Jeuken, L. J. C.; Beales, P. A., Durable proteo-hybrid vesicles for the extended functional lifetime of membrane proteins in bionanotechnology. Chemical Communications 2016.

26. Panneerselvam, K.; Lynge, M. E.; Riber, C. F.; Mena-Hernando, S.; Smith, A. A. A.; Goldie, K. N.; Zelikin, A. N.; Stadler, B., Phospholipid-polymer amphiphile hybrid assemblies and their interaction with macrophages. Biomicrofluidics 2015, 9 (5).

27. Schulz, M.; Olubummo, A.; Bacia, K.; Binder, W. H., Lateral surface engineering of hybrid lipidBCP vesicles and selective nanoparticle embedding. Soft Matter 2014, 10 (6), 831-839.

28. Pedersen, J. S., Neutron, X-rays and Light. Scattering Methods Applied to Soft Condensed Matter (North-Holland Delta Series) Elsevier ed.; Elsevier: Amsterdam, 2002; p 103-124.

29. Heberle, F. A.; Doktorova, M.; Goh, S. L.; Standaert, R. F.; Katsaras, J.; Feigenson, G. W., Hybrid and Nonhybrid Lipids Exert Common Effects on Membrane Raft Size and Morphology. Journal of the American Chemical Society 2013, 135 (40), 14932-14935.

30. Anghel, V. N. P.; Kucerka, N.; Pencer, J.; Katsaras, J., Scattering from laterally heterogeneous vesicles. II. The form factor. Journal of Applied Crystallography 2007, 40, 513-525.
31. Heberle, F. A.; Anghel, V. N. P.; Katsaras, J., Scattering from phase-separated vesicles. I. An analytical form factor for multiple static domains. Journal of Applied Crystallography 2015, 48, 13911404.

32. Bergstrom, L. M.; Skoglund, S.; Edwards, K.; Eriksson, J.; Grillo, I., Spontaneous Transformations between Surfactant Bilayers of Different Topologies Observed in Mixtures of Sodium Octyl Sulfate and Hexadecyltrimethylammonium Bromide. Langmuir 2014, 30 (14), 3928-3938.

33. Pedersen, J. S.; Posselt, D.; Mortensen, K. J., Analytical treatment of the resolution function for small-angle scattering. Journal of Applied Crystallography 1990, 23, 321-333.

34. Mildner, D. R. M.; Carpenter, Optimization of the experimental resolution for small-angle scattering. Journal of Applied Crystallography 1984, 17, 249-256. 
FOR TABLE OF CONTENTS USE ONLY",

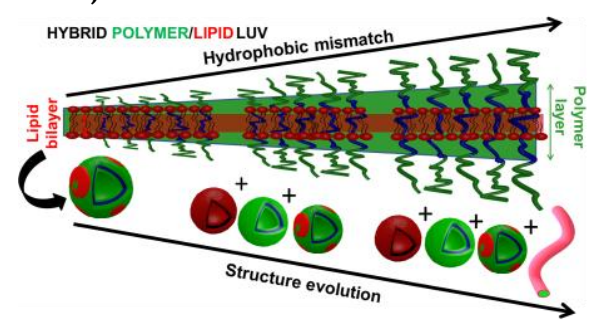

Mixing block copolymers with phospholipids at the nanoscale: from hybrid polymer/lipid worm-like micelles to vesicles presenting lipid nano-domains

T. P. Tuyen Dao, A. Brûlet, F. Fernandes, M. Er-Rafik, K. Ferji,R. Schweins, J-P. Chapel, A. Fedorov, M. Schmutz, M. Prieto, O. Sandre, J-F. Le Meins 